\title{
Estudio baritado del tubo digestivo alto en pacientes postoperados de cirugía bariátrica: Hallazgos normales y patológicos
}

Dres. José A. de Grazia $K^{(1)}$, Marcelo Godoy $Z^{(1)}$, Ignacia Torrealba $A^{(1)}$, Mauricio Guzmán $G^{(1)}$, Paula Csendes $G^{(2)}$, Attila Csendes $\mathrm{J}^{(3)}$.

1. Médico Becado de Radiología, Centro de Imagenología Hospital Clínico Universidad de Chile.

2. Unidad de Abdomen y Pelvis, Centro de Imagenología Hospital Clínico Universidad de Chile.

3. Departamento de Cirugía Hospital Clínico Universidad de Chile.

Upper gastrointestinal barium study in postoperative bariatric surgery patients: Normal and pathologic findings

Abstract: Objective. To evaluate a barium study of the upper gastrointestinal tract in early postoperative monitoring of bariatric surgery patients, recording the anatomical characteristics of the gastric remnant and the frequency of complications such as leakage of contrast and emptying difficulties. Materials and Methods. We retrospectively evaluated the barium study of the upper gastrointestinal tract performed in 168 patients after gastric bypass (GBP) and 98 patients after vertical subtotal gastrectomy (VSG). All evaluated examinations were performed in the first five days after surgery. Gastric remnant volume was registered in relation to the T12 vertebral body (only in GBP), the presence and site of contrast leakage and gastric remnant emptying difficulties. Results. The average age of patients undergoing GBP was 43 years, and 37 years for VSG. The average volume of gastric remnant of patients with GBP was 1.5 T12 vertebral bodies. Two GBP operated patients (1.2\%) had leakage of the contrast medium at the level of the gastroenteric anastomosis; in VSG operated patients the contrast leakage occurred in 7 cases (7.1\%), the most frequent site being the left side cephalic portion of the gastric remnant. All cases with contrast leakage were subsequently studied using computed tomography, finding the presence of wound dehiscence or fluid collections adjacent to the gastric remnant. Emptying difficulty was observed in 12 patients operated for GBP (7.1\%); there was no record of this complication in patients undergoing VSG. Conclusion. The upper gastrointestinal barium study is useful in patients after bariatric surgery to assess the early postoperative anatomy and volume of the gastric remnant, as well as to detect possible complications. It is a simple test, which should be applied as a routine control in these patients.

Keywords: Bariatric surgery, Gastric Bypass, Vertical subtotal gastrectomy, Upper gastrointestinal barium study.

Resumen: Objetivo. Evaluar el estudio baritado del tubo digestivo alto en el control postoperatorio precoz de pacientes operados de cirugía bariátrica, registrando las características anatómicas del remanente gástrico y la frecuencia de complicaciones como filtración del medio de contraste y dificultad al vaciamiento. Materiales y método. Se evaluó retrospectivamente el estudio baritado del tubo digestivo alto realizado en 168 pacientes postoperados de bypass gástrico (BPG) y 98 pacientes postoperados de gastrectomía subtotal vertical (GSV). Todos los exámenes evaluados fueron realizados en los primeros cinco días postcirugía. Se registró volumen del remanente gástrico en relación al cuerpo vertebral T12 (sólo en BPG), presencia y sitio de filtración del medio de contraste y dificultad al vaciamiento del remanente gástrico. Resultados. La edad promedio de los pacientes sometidos a BPG fue de 43 años y GSV 37 años. El volumen promedio del remanente gástrico de los pacientes con BPG fue 1,5 cuerpos vertebrales $T 12$. Dos pacientes operados de BPG $(1,2 \%)$ presentaron filtración de medio de contraste a nivel de la anastomosis gastro-enteral; en los pacientes operados de GSV la filtración del medio de contraste ocurrió en 7 casos (7,1\%), siendo el sitio más frecuente la porción cefálica lateral izquierda del remanente gástrico. Todos los casos de filtración de medio de contraste fueron posteriormente estudiados con tomografía computada, comprobándose la presencia de dehiscencia de la sutura o bien colecciones líquidas adyacentes al remanente gástrico. Se observó dificultad al vaciamiento en 12 pacientes operados de BPG (7,1\%); no se registró esta complicación en los pacientes operados de GSV. Conclusión. El 
estudio baritado del tubo digestivo alto es útil en pacientes postoperados de cirugía bariátrica para conocer la anatomía y el volumen del remanente gástrico en el postoperatorio precoz, así como también para detectar posibles complicaciones. Es un examen sencillo, que debiese aplicarse como control rutinario en estos pacientes. Palabras clave: Cirugía bariátrica, bypass gástrico, gastrectomía subtotal vertical, estudio baritado del tubo digestivo alto.

de Grazia, et al. Estudio Baritado del Tubo Digestivo Alto en Pacientes Postoperados de Cirugía Bariátrica: Hallazgos Normales y Patológicos. Rev Radiol 2013; 19(2): 53-59.

Trabajo presentado en el Congreso Chileno de Radiología 2012 y Primera Jornada Chileno-Española de Radiología, octubre de 2012. Enviado: 29- 03-2013. Aceptado para publicación: 28- 04-2013.

\section{Introducción}

La obesidad constituye una epidemia mundial en el siglo XXI. Chile no está ajeno a esta realidad, con cifras reportadas de obesidad en hombres y mujeres de 19 y $25 \%$ respectivamente, destacando cifras de obesidad mórbida de 0,2 y $2,3 \%$ respectivamente ${ }^{(1)}$. Esto se traduce en un número de cerca de 150.000 obesos mórbidos en nuestro país(2).

Los pacientes que han fallado en alcanzar o mantener la pérdida de peso con tratamiento médico supervisado son candidatos a cirugía bariátrica ${ }^{(3,4)}$. La cirugía bariátrica permite considerable pérdida de peso, mantención prolongada del nuevo peso alcanzado y control o incluso reversión de algunos de los problemas de salud asociados a la obesidad( ${ }^{(5,6)}$.

Las tasas de morbi-mortalidad descritas a nivel nacional para esta cirugía son bajas ${ }^{(7)}$. Las principales complicaciones postoperatorias locales tempranas incluyen filtración de la anastomosis o suturas y estenosis o dificultad al vaciamiento gástrico. La tasa reportada en la literatura para filtración de la anastomosis o suturas en pacientes operados de bypass gástrico (BPG) es $3,4 \%{ }^{(8)}$, siendo la localización más frecuente la anastomosis gastro-enteral ${ }^{(8)}$, con buena respuesta al tratamiento médico conservador ${ }^{(8,9)}$. En el caso de la gastrectomía subtotal vertical (GSV), la cifra de filtración de la sutura del remanente gástrico es de $4,7 \%{ }^{(10)}$, manejándose las filtraciones precoces usualmente en forma quirúrgica ${ }^{(10)}$. La literatura internacional describe tasas de incidencia de dificultad al vaciamiento del remanente gástrico de 3,6 y 3,0\% para BPG y GSV respectivamente(11). Todas estas complicaciones, a pesar de ser poco frecuentes, pueden ser detectadas con un estudio baritado del tubo digestivo alto.

Existe controversia en la literatura respecto a la utilidad del estudio baritado del tubo digestivo alto realizado en forma rutinaria durante el postoperatorio precoz de pacientes operados de cirugía bariátrica. Algunos autores lo apoyan, argumentando que tiene una buena capacidad para detectar las complicaciones ${ }^{(11,12)}$, mientras otros argumentan que tiene baja sensibilidad y alta tasa de falsos negativos y falsos positivos $^{(13,14)}$. Otro punto a favor es que permite contar con un registro a "tiempo cero" del volumen del remanente gástrico.

El objetivo del presente estudio es describir los hallazgos normales y patológicos del estudio baritado del tubo digestivo alto realizado en forma rutinaria en el control postoperatorio precoz de pacientes operados de cirugía bariátrica (BPG y GSV), registrando las características anatómicas del remanente gástrico y la frecuencia de complicaciones como filtración del medio de contraste y dificultad al vaciamiento del remanente gástrico.

\section{Materiales y método}

Se evaluó en forma retrospectiva el estudio baritado de tubo digestivo alto realizado en 168 pacientes postoperados de bypass gástrico con reconstrucción en $Y$ de Roux (BPG) y 98 pacientes postoperados de gastrectomía subtotal vertical "en manga" (GSV). Todas las cirugías fueron laparoscópicas, ninguna correspondiendo a una reoperación de cirugía bariátrica. Los estudios baritados del tubo digestivo alto fueron realizados en sala telecomandada, en los primeros cinco días postcirugía.

El protocolo de estudio incluyó el uso de cantidades variables de suspensión baritada (sin superar los 100 $\mathrm{cc}$ ), en dosis suficiente como para opacificar esófago, remanente gástrico y porción proximal de intestino delgado. El medio de contraste fue administrado en sorbos intermitentes antes de obtener cada proyección radiográfica. Se obtuvo una proyección $\mathrm{AP}$ de pie de la región epigástrica, previo a la administración de medio de contraste, para identificar suturas y clips metálicos en el área quirúrgica. Luego, se obtuvieron proyecciones en decúbito supino, decúbito lateral derecho e izquierdo y decúbito prono posterior a la administración de suspensión baritada vía oral. Finalmente se obtuvo una proyección AP de pie panorámica del abdomen. En el caso de los pacientes operados de BPG, se obtuvo una radiografía AP de abdomen complementaria luego de dos horas, para documentar la llegada del medio de contraste al colon y descartar dilatación patológica de asas intestinales. Las figuras 1 y 2 muestran protocolos de estudio normal para BPG y GSV respectivamente. 

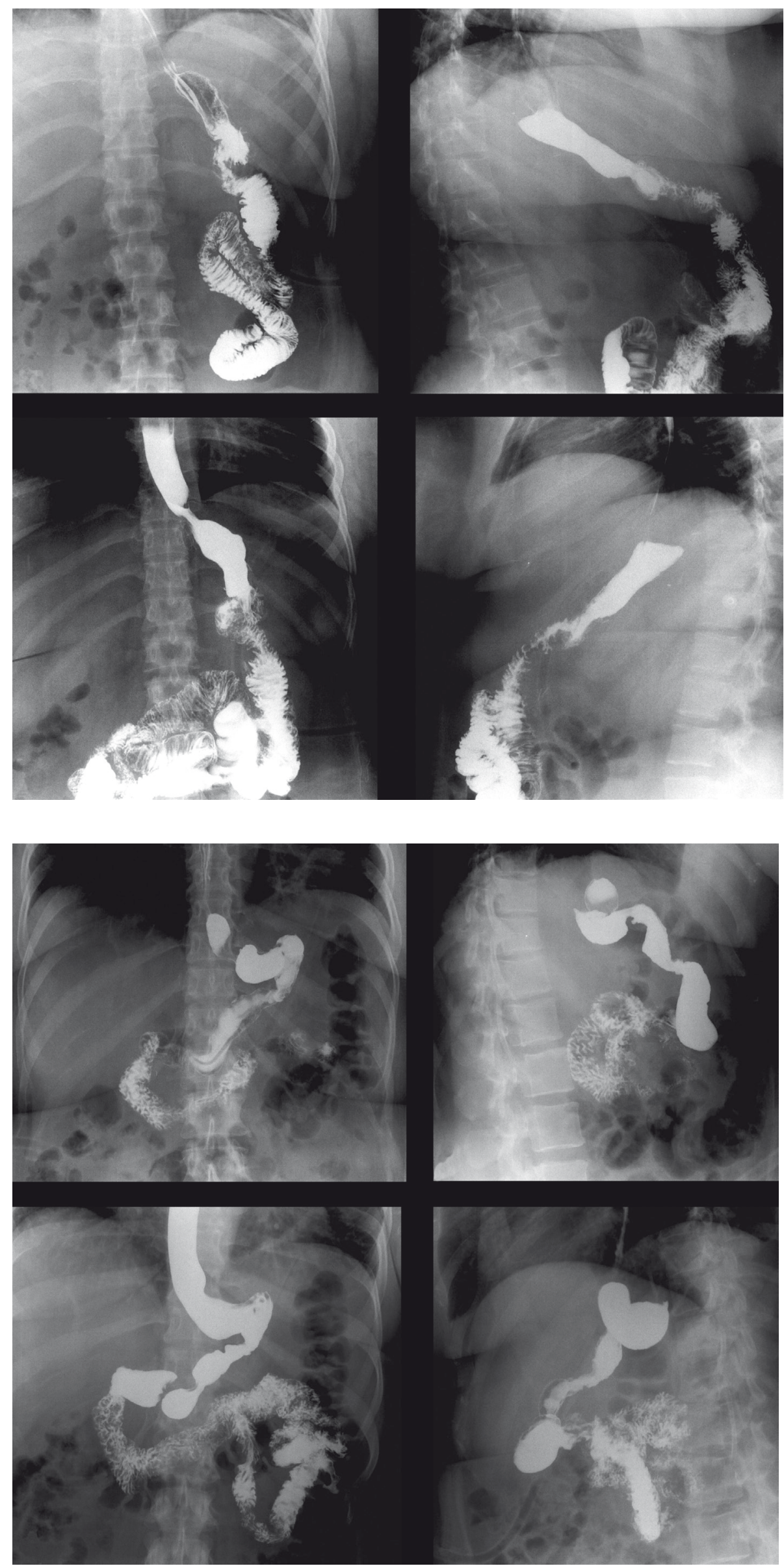

Figura 1. Estudio baritado del tubo digestivo alto en paciente con bypass gástrico

Proyecciones obtenidas luego de la administración de suspensión baritada vía oral. A: decúbito supino; $B$ : decúbito lateral izquierdo; C; decúbito prono; $D$; decúbito lateral derecho. No se incluyeron las proyecciones $A P$ de pie de la región epigástrica previo a la administración de medio de contraste, AP de pie panórámica del abdomen luego de la administración de medio de contraste, ni AP de abdomen complementaria a las 2 horas. El volumen del remanente gástrico de este paciente fue estimado en 2 cuerpos vertebrales T12. No se evidencian filtraciones ni dificultad de vaciamiento del remanente gástrico.

Figura 2. Estudio baritado del tubo digestivo alto en paciente con gastrectomía subtotal vertical Proyecciones obtenidas luego de la administración de suspensión baritada vía oral. A: decúbito supino; $B$ : decúbito lateral izquierdo; $C$; decúbito prono; $D$; decúbito lateral derecho. No se incluyeron las proyecciones $A P$ de pie de la región epigástrica previo a la administración de medio de contraste ni AP de pie panórámica del abdomen luego de la administración de medio de contraste. No se evidencian filtraciones ni dificultad de vaciamiento del remanente gástrico. 
En cada paciente se registró:

Volumen del remanente gástrico en relación al cuerpo vertebral T12 (sólo en BPG), realizado mediante una estimación subjetiva por parte del observador.

Presencia de filtración del medio de contraste y sitio de ésta en caso de haberla, definida como una extravasación de medio de contraste por fuera de los límites del remanente gástrico, ya sea contenida o con difusión hacia la cavidad peritoneal o los tubos de drenaje.

Dificultad al vaciamiento del remanente gástrico, definida como retención del medio de contraste en esófago y remanente gástrico por un tiempo mayor a un minuto, sin avance hacia distal.

En todos los casos en que se registró filtración del medio de contraste, se revisó la tomografía computada realizada posteriormente al paciente, para consignar hallazgos directos o indirectos compatibles con dehiscencia de la anastomosis o sutura.

\section{Resultados}

La edad promedio de los pacientes sometidos a BPG fue de 43 años y GSV 37 años. El IMC promedio para ambos grupos fue 43,7 y $38,0 \mathrm{Kg} . / \mathrm{m}^{2}$ respectivamente.

El volumen promedio del remanente gástrico de los pacientes con BPG fue 1,5 cuerpos vertebrales T12, lo que equivale a 40 cc según estudios morfométricos de volumetría de cuerpos vertebrales ${ }^{(15)}$.

Dos pacientes operados de BPG (1,2\%) presentaron filtración de medio de contraste a nivel de la anastomosis gastro-enteral. En los pacientes operados de GSV la filtración del medio de contraste ocurrió en 7 casos (7,1\%), presentándose ésta a nivel de la línea de sutura en la curvatura mayor, siendo en 6 de los 7 casos la porción cefálica de esta línea de sutura la que filtraba (Figura 3) y en el caso restante la filtración era más baja, localizándose en la línea de sutura a nivel del cuerpo gástrico (Figura 4). Todas las filtraciones registradas tanto en BPG como GSV fueron de bajo volumen, permaneciendo el medio de contraste contenido en las proximidades del remanente gástrico, a excepción de un paciente con GSV, en el cual la filtración fue de mayor volumen, con difusión hacia la cavidad peritoneal y por alrededor de los tubos de drenaje (Figura 4).

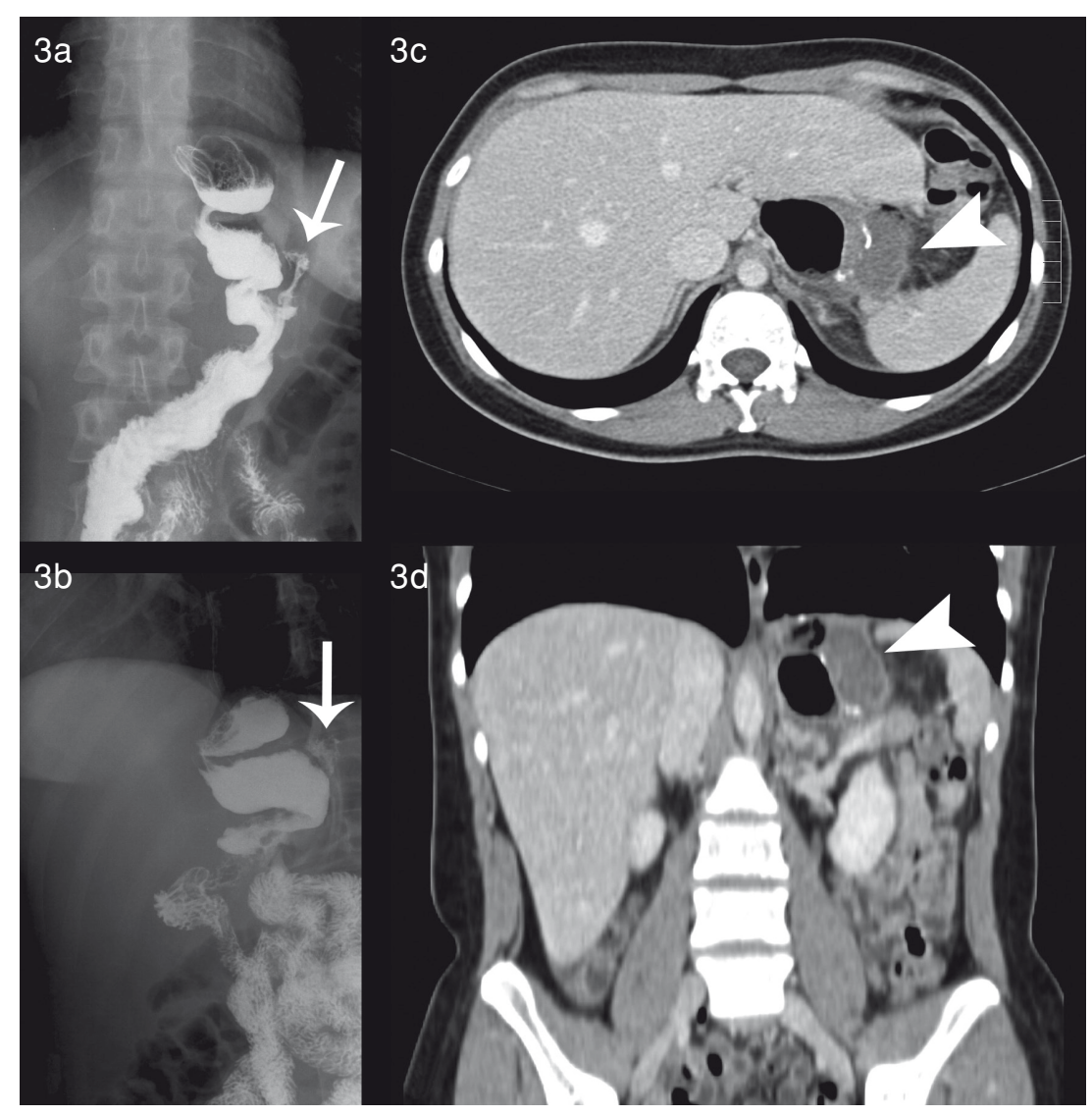

Figura 3. Filtración de la sutura del remanente gástrico en paciente operado de GSV.

Estudio baritado del tubo digestivo alto: a: proyección en decúbito supino; b: proyección en decúbito lateral derecho. La flecha señala la filtración de medio de contraste en el aspecto superior de la línea de sutura en la curvatura mayor.

Tomografía computada realizada al día siguiente; c: corte axial en fase porto-venosa; d: reconstrucción coronal en fase porto-venosa. La cabeza de flecha señala una colección líquida perigástrica, adyacente a la línea de sutura en la curvatura mayor del remanente gástrico. 


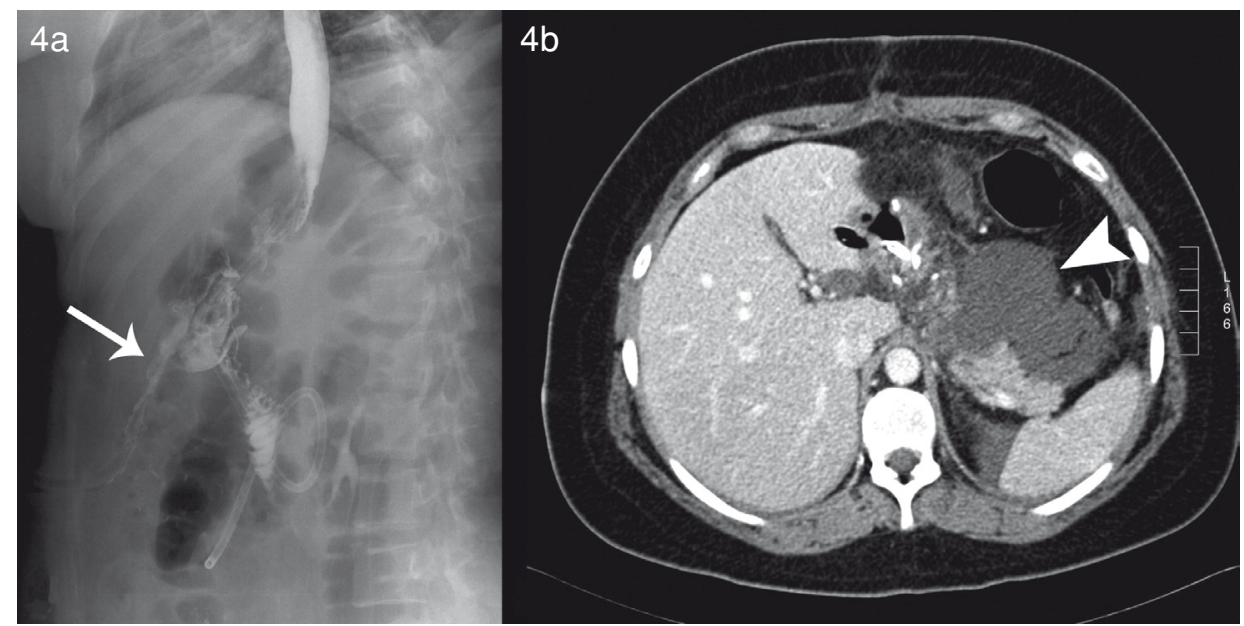

Figura 4. Filtración de la sutura del remanente gástrico en paciente operado de GSV

Estudio baritado del tubo digestivo alto: a) proyección en decúbito lateral derecho. La flecha señala la filtración de medio de contraste a nivel del cuerpo gastrico en la línea de sutura en la curvatura mayor, con difusión del medio de contraste hacia la cavidad peritoneal y opacificación del tubo de drenaje.

Tomografía computada realizada al día siguiente: b) corte axial en fase porto-venosa. La cabeza de flecha señala una colección líquida adyacente a la línea de sutura en la curvatura mayor del cuerpo del remanente gástrico, existe además escaso líquido libre periesplénico.

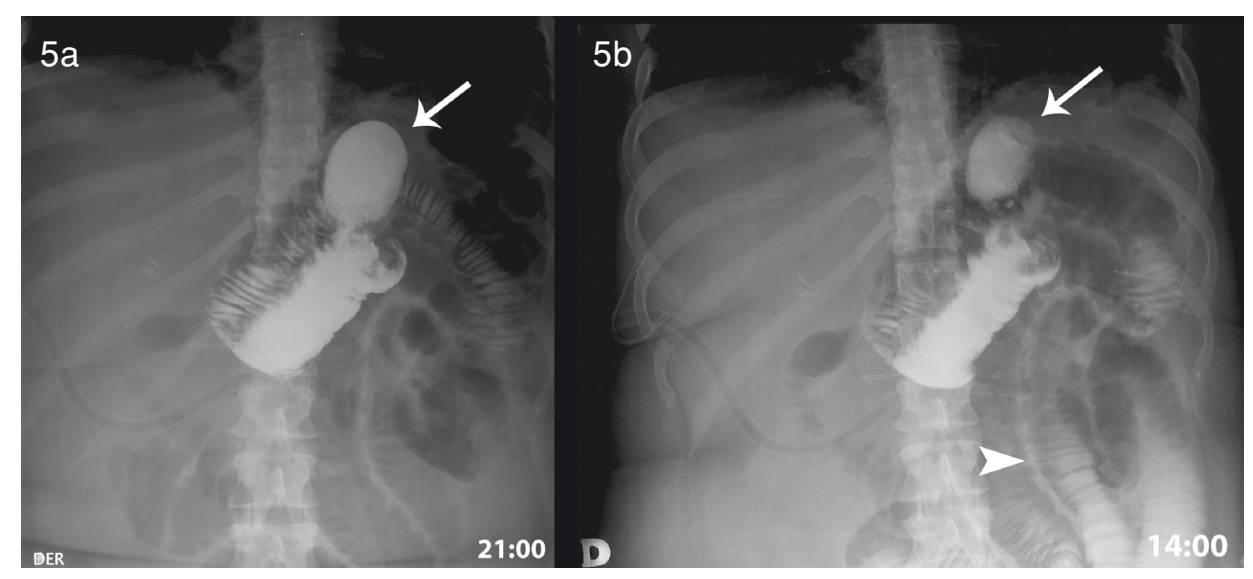

Figura 5. Dificultad al vaciamiento del remanente gástrico en paciente operado de GSV. Estudio baritado del tubo digestivo alto: a) proyección en decúbito supino obtenida a las 21:00 hrs, que muestra un remanente gástrico distendido, con escaso paso del medio de contraste a través de anastomosis gastro-enteral hacia asas de intestino delgado que se observan dilatadas: b) proyección en decúbito supino obtenida a las 14:00 hrs del día siguiente, que muestra perisitencia parcial de medio de contraste en el remanente gástrico, con escaso avance de éste a través del intestino delgado, el cual se observa dilatado (cabeza de flecha).

En todos los casos en que se registró filtración del medio de contraste se revisó la tomografía computada realizada posteriormente al paciente, comprobándose la presencia de colecciones líquidas adyacentes al remanente gástrico en todos los casos (Figuras 3 y 4), lográndose observar además la dehiscencia de la sutura en un caso de GSV.

Se observó dificultad al vaciamiento del remanente gástrico en 12 pacientes operados de BPG $(7,1 \%)$ (figura 5). No se registró esta complicación en los pacientes operados de GSV.

\section{Discusión}

El estudio baritado del tubo digestivo alto realizado en forma rutinaria durante el postoperatorio precoz a pacientes sometidos a cirugía bariátrica permite contar con un registro a "tiempo cero" del volumen del remanente gástrico, para poder comparar con exámenes posteriores y además predecir el éxito en la reducción del exceso de peso del paciente. En nuestro trabajo, el volumen promedio del remanente gástrico de los pacientes con BPG fue 1,5 cuerpos vertebrales T12, lo que equivale a $40 \mathrm{cc}$. Estudios 
realizados con exámenes contrastados del tubo digestivo alto indican que el volumen promedio del remanente gástrico en el postoperatorio precoz de pacientes con BPG es entre 30 y $60 \mathrm{cc}^{(14,15)}$. La literatura internacional es controversial en cuanto a la asociación entre menor volumen del remanente gástrico en el postoperatorio precoz y mayor pérdida de exceso de peso del paciente con BPG a corto y mediano plazo, con estudios que están a favor de esta asociación ${ }^{(16)}$ y otros en contra ${ }^{(17)}$. No realizamos estimación subjetiva del volumen del remanente gástrico en relación al cuerpo vertebral T12 en los pacientes con GSV, ya que resulta complejo hacer la estimación subjetiva del volumen de una estructura tubular y la variabilidad interobservador es muy alta.

La tasa obtenida de filtración de medio de contraste en pacientes operados de BPG fue de 1,2\%, valor similar a lo descrito en la literatura( ${ }^{(8,9)}$. En los 2 casos encontrados la filtración ocurrió a nivel de la anastomosis gastro-enteral, lo que resulta compatible con los datos reportados en estudios previos, que señalan que más del $50 \%$ de las filtraciones en pacientes operados de BPG ocurren a este nivel ${ }^{(8)}$. En términos generales, las filtraciones precoces pueden ser manejadas con manejo médico conservador (nutrición enteral con sonda naso-yeyunal, mantención de tubos de drenaje o drenaje percutáneo de colecciones líquidas por radiología intervencional, antibióticos en caso de fiebre), con buena respuesta ${ }^{(8,9)}$, debido a que no hay producción de ácido en el pequeño remanente gástrico y tampoco hay reflujo intestinal debido a la longitud larga del asa de Roux o alimentaria( ${ }^{(9)}$. En el caso de los pacientes operados de GSV la tasa de filtración de medio de contraste fue baja $(7,1 \%)$, pero algo más alta que la reportada en estudios previos ${ }^{(10)}$. La localización de la filtración fue mayoritariamente en la porción cefálica de la línea de sutura en la curvatura mayor, lo cual resulta similar a lo descrito en la literatura 10, situación que posiblemente está determinada por el acceso más dificultoso del stapler por vía laparoscópica hasta ese punto. Las filtraciones precoces en pacientes operados de GSV son manejadas habitualmente en forma quirúrgica ${ }^{(10)}$.

Respecto a la dificultad al vaciamiento del remanente gástrico, la tasa obtenida fue $7,1 \%$ en pacientes operados de BPG, sin registrarse esta complicación en los pacientes operados de GSV. Este hallazgo es común de observar en el postoperatorio precoz de estos pacientes y muchas veces es resultado del edema y cambios postoperatorios a nivel de la anastomosis gastro-enteral y/o entero-enteral o bien íleo postoperatorio, sin traducir una real estenosis de las anastomosis. Un estudio realizado con endoscopía un mes después de la cirugía demuestra que un $36 \%$ de los pacientes operados de BPG laparoscópico presenta estenosis de la anastomosis gastro-enteral, siendo la mayoría leve o moderada (diámetro mayor o igual a $5 \mathrm{~mm}$ ) que no requirieron dilatación endoscópica y presentaron control endoscópico normal a los 17 meses $^{(18)}$.

Finalmente, en relación a la controversia existente en la literatura respecto a la utilidad del estudio baritado del tubo digestivo alto realizado en forma rutinaria durante el postoperatorio precoz de pacientes operados de cirugía bariátrica, podemos señalar que en nuestro estudio este examen fue una herramienta útil, que nos aportó un registro a "tiempo cero" del volumen del remanente gástrico y nos permitió detectar las complicaciones habituales de esta cirugía con un buen correlato con la tomografía computada. No realizamos cálculos de las propiedades diagnósticas del examen (sensibilidad, especificidad y valores predictivos), pues consideramos que no disponíamos de un gold standard adecuado para comparar, pues no todos los paciente evaluados con estudio baritado del tubo digestivo alto fueron posteriormente evaluados con tomografía computada.

\section{Conclusión}

El estudio baritado del tubo digestivo alto es útil en pacientes postoperados de cirugía bariátrica para conocer la anatomía y el volumen del remanente gástrico en el postoperatorio precoz ("tiempo cero"), así como también para detectar posibles complicaciones. Es un examen sencillo, que debiese aplicarse como control rutinario en estos pacientes.

\section{Bibliografía}

1. Ministerio de Salud, Chile. Encuesta Nacional de Salud. 2003.

2. Pizarro T. Estadísticas nutricionales del Ministerio de Salud, Chile. 2003.

3. Álvarez-Cordero R. Treatment of clinically severe obesity, a public health problem. World J Surg 1998; 22(9): 905-906.

4. Martin LF, White S, Lindstrom W Jr. Cost-benefit analysis for the treatment of severe obesity. World J Surg 1998; 22(9):1008-1017.

5. Pories WJ, Swanson MS, MacDonald KG, Long SB, Morris PG, Brown BM, et al. Who would have thought it? An operation proves the most effective therapy for adult onset diabetes mellitus. Ann Surg 1995; 222(3): 339-352.

7. Baltasar A, Serra C, Pérez N, Bou R, Bengoechea M, Ferri L. Laparoscopic sleeve gastrectomy: a multipopouse bariatric operation. Obes Surg 2005; 15(8): 1124-1128.

8. Csendes A, Maluenda F. Morbimortalidad de la cirugía bariátrica. Experiencia chilena en 10 instituciones de salud. Rev Chil Cir 2006; 58(3): 208-212.

9. Csendes A, Burgos AM, Braghetto I. Classification and management of leaks after gastric bypass for patients with morbid obesity: a prospective study for 60 patients. Obes Surg 2012; 22(6): 855-862.

10. Csendes A, Burdiles P, Burgos AM, Maluenda F, Díaz JC. Conservative management of anastomotic leaks 
after 557 open gastric bypasses. Obes Surg 2005; 15(9): 1252-1256.

11. Csendes A, Braghetto I, León P, Burgos AM. Management of leaks after laparoscopic sleeve gastrectomy in patients with obesity. J Gastrointest Surg 2010; 14(9): 1343-1348.

12. Toppino M, Cesarani F, Comba A, Denegri F, Mistrangelo M, Gandini G, et al. The role of early radiological studies after gastric bariatric surgery. Obes Surg 2001; 11(4): 447-454.

13. Raman R, Raman B, Raman P, Rossiter S, Curet MJ, Mindelzun R, et al. Abnormal findings on routine upper Gl series following laparoscopic Roux-en-Y gastric bypass. Obes Surg 2007; 17(3): 311-316.

14. Carter JT, Tafreshian S, Campos GM, Tiwari U, Herbella F, Cello JP. Routine upper GI series after gastric bypass does not reliably identify anastomotic leaks or predict stricture formation. Surg Endosc 2007; 21(12): 2172-2177.

15. Doraiswamy A, Rasmussen JJ, Pierce J, Fuller W, Ali
MR. The utility of routine postoperative upper GI series following laparoscopic gastric bypass. Surg Endosc 2007; 21(12): 2159-2162.

16. Limthongkul W, Karaikovic EE, Savage JW, Markovic A. Volumetric analysis of thoracic and lumbar vertebral bodies. The Spine Journal 2010; 10(2): 153-158.

17. Roberts K, Duffy A, Kaufman J, Burrell M, Dziura J, Bell R. Size matters: gastric pouch size correlates with weight loss after laparoscopic Roux-en-Y gastric bypass. Surg Endosc 2007; 21(8): 1397-1402.

18. Nishie A, Brown B, Barloon T, Kuehn D, Samuel I. Comparison of size of proximal gastric pouch and shortterm weight loss following routine upper gastrointestinal contrast study after laparoscopic Roux-en-Y gastric bypass. Obes Surg 2007; 17(9): 1183-1188.

19. Csendes A, Burgos AM, Burdiles P. Incidence of anastomotic strictures after laparoscopic bypass: a prospective consecutive routine endoscopic study 1 month and 17 months after surgery in 441 patients with morbid obesity. Obes Surg 2009; 19(3): 269-273. 